\title{
Linolenic fatty acid hydroperoxide acts as biocide on plant pathogenic bacteria: Biophysical investigation of the mode of action
}

\author{
Deboever Estelle ${ }^{\mathrm{a}, \mathrm{b}, *}$, Lins Laurence ${ }^{\mathrm{a}}$, Ongena Marc ${ }^{\mathrm{c}}$, De Clerck Caroline ${ }^{\mathrm{d}, \mathrm{e}}$, Deleu Magali ${ }^{\mathrm{a}, 1}$, \\ Fauconnier Marie-Laure ${ }^{\mathrm{b}, 1}$ \\ ${ }^{a}$ Laboratoire de Biophysique Moléculaire aux Interfaces (LBMI), Gembloux Agro-Bio Tech, Université de Liège, 2, Passage des Déportés, B-5030 Gembloux, Belgium \\ ${ }^{\mathrm{b}}$ Laboratoire de Chimie des Molécules Naturelles (LCMN), Gembloux Agro-Bio Tech, Université de Liège, 2, Passage des Déportés, B-5030 Gembloux, Belgium \\ ${ }^{\mathrm{c}}$ Microbial Processes and Interactions (MiPI), Gembloux Agro-Bio Tech, Université de Liège, 2, Passage des Déportés, B-5030 Gembloux, Belgium \\ ${ }^{\mathrm{d}}$ Agriculture Is Life, Gembloux Agro-Bio Tech, Université de Liège, 2, Passage des Déportés, B-5030 Gembloux, Belgium \\ ${ }^{\mathrm{e}}$ Laboratoire de Phytopathologie Intégrée et Urbaine, Gembloux Agro-Bio Tech, Université de Liège, 2, Passage des Déportés, B-5030 Gembloux, Belgium
}

\section{A R T I C L E I N F O}

\section{Keywords:}

Linolenic fatty acid hydroperoxide

13-HPOT

Oxylipins

Antibacterial activity

Membrane interaction

\begin{abstract}
A B S T R A C T
Fatty acid hydroperoxides (HPO) are free phyto-oxylipins known for their crucial role as signalling molecules during plant defense mechanisms. They were also demonstrated to have direct biocidal activities against plant pathogens including gram negative bacteria. In the present work, the biocidal effect of one linolenic fatty acid hydroperoxide, namely 13-HPOT has been investigated on three plant pathogen gram negative bacteria: Pectobacterium carotovorum, Pseudomonas syringae and Xanthomonas translucens. We showed that 13-HPOT has a strong dose response effect on those phytopathogens.

In a second part, the molecular mechanism behind the antibacterial effect of 13-HPOT was investigated at a molecular level using an integrative biophysical approach combining in vitro and in silico methods. Since other antimicrobial amphiphilic molecules have been shown to target the lipid membrane of the organisms they act on, we focused our study on the interaction of 13-HPOT with biomimetic membranes. In a first step, we hypothesized that the inner membrane of the bacteria was the main site of action of 13-HPOT and hence we used lipids representative of this membrane to form our models.

Our results indicated that 13-HPOT can interact with the lipid representative of the inner bacterial plasma membrane. A strong membrane insertion is suggested but no major permeabilization of the membrane is observed. Phosphatidylethanolamine (PE) and cardiolipin (CL), present in the bacterial plasma membrane, appear to play important roles in this interaction. We suggest that the mode of action of 13-HPOT should involve either subtle changes in membrane properties, such as its lateral organization and distribution, and/or interactions with membrane proteins.
\end{abstract}

\section{Introduction}

Plant oxylipins are produced under a wide range of stress conditions. They are implicated in physiological processes (plant growth, development and senescence) or response to stress (stimulation of plant defenses, defense gene expression, hypersensitive response, etc.) [1,2]. Oxylipin is the common name for oxidized polyunsaturated fatty acids (PUFAs) and their resulting metabolites. Their synthesis starts with the oxidation of PUFAs chemically (autooxidation) or enzymatically via the action of lipoxygenases (LOXs, E.C 1.13.11.12) or $\alpha$-dioxygenase (E.C

\footnotetext{
Abbreviations: 13-HPOD, 13-hydroperoxi-9,11,15-octadecadienoic acid; 13-HPOT, 13-hydroperoxi-9,11,15-octadecatrienoic acid; BM, Big Monolayer; BPM, bac-

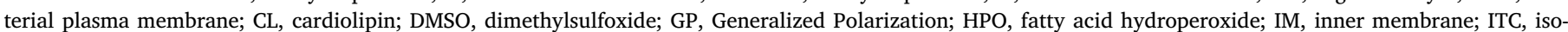

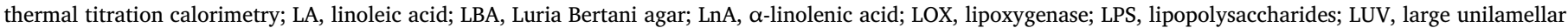

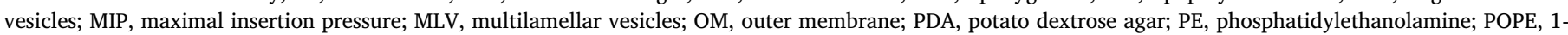

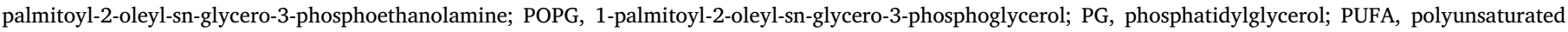
fatty acids; SUV, small unilamellar vesicles; TRIS, tri(hydroxymethyl)aminomethane

*Corresponding author at: Laboratoire de Biophysique Moléculaire aux Interfaces (LBMI), Gembloux Agro-Bio Tech, Université de Liège, 2, Passage des Déportés, B-5030 Gembloux, Belgium.

E-mail address: estelle.deboever@doct.uliege.be (D. Estelle).

${ }^{1}$ These authors have equally contributed to this work and must be considered as co-last authors.
} 
1.13.11) [1,3-9]. LOXs are ubiquitous enzymes found in both animals and plants $[1,10]$. In plants, LOXs convert mainly 1-4 pentadiene structure into fatty acid hydroperoxides (HPO), such as 9 or 13-hydroperoxi-9,11,15-octadecatrienoic acid (HPOT) from $\alpha$-linolenic acid (LnA, C18:3) and 9 or 13-hydroperoxi-9,11-octadecadienoic acid (HPOD) from linoleic acid (LA, C18:2) [11-13]. These can further be converted into several biologically active compounds varying in their chemical structures $[1,4,7]$, but a part of HPOs is also found untransformed in plants [14-16].

Recently, plant oxylipins were described as biocide compounds. Prost et al. have tested, in vitro, different oxylipins by exogenous application on several plant pathogens. Especially, key intermediate oxylipins (HPOD and HPOT) were shown to be crucial agents in the plant direct defense against fungi and bacteria $[17,18]$. Previous studies on bacteria only focused on 13-HPOD and on one concentration $(100 \mu \mathrm{M})$ [17]. Antimicrobial properties of 13-HPOT were investigated on the yeast model Yarrowia lipolytica, a rare opportunistic fungal pathogen [19], and they were shown to be related to strong interactions of this compound with membrane lipids [20]. Nonetheless, the complete mechanism involved in their perception by the pathogen cell surface is far from being known [21]. As many antibacterial molecules have proved their efficiency through specific membrane interactions [22,23], such behavior can be expected with phyto-oxylipins including 13HPOT. Indeed, in a previous work based on HPOs interactions with plant biomimetic plasma membranes, we have demonstrated that 13HPOT has more affinity for model bilayer and displays a faster adsorption kinetic as compared to 13-HPOD [24]. The presence of three double bonds seems to be the key structural trait as 13-HPOT has a higher biocidal effect in in vitro assays [17]. However, there is still no study on the HPOT-bacterial membrane interactions.

A large part of phytopathogenic bacteria belongs to the Gram-negative group, with Agrobacterium, Pectobacterium, Pseudomonas, Xanthomonas and Xyllela being responsible for most diseases in the field [22]. The first objective of this study was to analyze the antibacterial properties of 13-HPOT on three pathogens of agronomic interest: Pectobacterium carotovorum, Pseudomonas syringae p.v. syringae DC3000 and Xanthomonas translucens p.v. translucens.

One target of 13-HPOT could be the bacterial membrane. Few years ago, interaction and disruption of the plasma membrane appeared as a new track for antibacterial therapy in Humans [25-27]. Less known than in eukaryotes, the composition of bacteria membranes is very diversified, especially for Gram-negative bacteria. They possess two distinct membranes: the outer membrane (OM) and the inner membrane (IM) separated by a thin layer of peptidoglycan [28]. The OM is mainly composed of lipopolysaccharides (LPS) and some glycerophospholipids, whereas the IM is fully composed of glycerophospholipids (mainly phosphatidylethanolamine (PE), phosphatidylglycerol (PG) and cardiolipin (CL)) [23,28-33]. Proteins have always been considered as the main actors of physiological functions across biological membranes. But in recent years, numerous studies have focused on the complexity of the membranes and, in particular, the essential role of lipids in their organization and intrinsic properties. New perspectives have appeared on the effect of amphiphilic drugs on lipid-protein interactions, with direct consequences on membrane lipid domains $[23,34,35]$. In turn, these modifications influence the localization/activity of proteins, particularly highly sensitive to changes in the IM [28]. Many amphiphilic molecules have shown their capacity to interact specifically with the lipids constituting the plasma membrane and, in certain cases, to influence its properties [23,34,36-38]. Especially, some molecules extracted from plants or of plant origin would derive their multiple effects via their ability to interact with the lipid part of the membranes $[39,40]$. Such interactions are likely to have a significant impact on the functioning of the membrane and could explain the mode of action or the side effects of certain compounds. In this paper, we have decided to focus on the interaction of 13-HPOT with IM, since this molecule has shown strong interactions with plant glycerolipids that are also the IM main components but with different lipid head natures. Furthermore, other small amphipiles, such as neamine derivatives, are able to interact both with OM and IM $[23,37,41]$. The IM can thus be one of the privileged target for antibacterial amphiphilic molecules. In order to investigate the antibacterial mechanisms at the molecular level, we have studied the interaction between 13-HPOT and bacterial plasma membrane (BPM) lipids using complementary in vitro and in silico biophysical approaches. Particularly, we have explored the interaction of 13HPOT with lipid models mimicking the IM of Pseudomonas sp. based on the lipid composition described in the literature $[23,37,42]$ with a special focus on the lipid specificity of the interaction and its potential effects on BPM lipid organization.

Overall, our results give valuable insights to discuss how the interaction mechanism can be related to the in vitro biocidal assays.

\section{Materials and methods}

\subsection{Materials}

As described in our previous works [24,43], 13-HPOT was enzymatically synthetized from the reaction of LOX-1 on linolenic acid. The purity (higher than 98\%) was checked by high-performance liquid chromatography. The bacterial strains Pectobacterium carotovorum and Pseudomonas syringae strain DC3000 were supplied by DSMZ-German Collection, Leibniz Institute (Germany) and Laboratory Stress, Defenses and Plant Reproduction, Reims University (France; Dr. Stephan Dorey) respectively. Xanthomonas translucens pv. translucens and Fusarium oxysporum f.sp. radices-lycopersici were supplied by BCCM Laboratory for Microbiology, University of Gent (Belgium). All bacterial strains were grown at $28{ }^{\circ} \mathrm{C}$ on LBA Petri dishes for at least $24 \mathrm{~h}$.

1-palmitoyl-2-oleyl-sn-glycero-3-phosphoethanolamine (POPE), 1palmitoyl-2-oleyl-sn-glycero-3-phosphoglycerol (POPG), heart bovine cardiolipin (CL), lipoxidase from Glycine max (soybean) type I-B (LOX1 ), the linoleic and $\alpha$ - linolenic acids, Luria Bertani Agar (LBA) and the potato dextrose agar (PDA) were purchased from Sigma-Aldrich (Belgium). Chloroform and methanol were both purchased from Scharlau Lab Co. Dimethylsulfoxide (DMSO) and tri(hydroxymethyl) aminomethane (TRIS) were provided by Sigma Chemical. The ultrapure water was produced by a Millipore system available in our laboratory, the resistivity was $18.2 \Omega \mathrm{cm}$.

\subsection{Biocidal assays}

In vitro antibacterial activity of 13-HPOT was evaluated in a microspectrophotometric assay, based on [17]. Bacteria were grown in sterile, flat-bottom, 96-well microplates, sealed with Parafilm, in a final volume of $100 \mu \mathrm{L}$ of LB liquid medium. They were grown at $28{ }^{\circ} \mathrm{C}$ starting with approximately 2,000 bacteria per well. 13-HPOT was dissolved in $\mathrm{H}_{2} \mathrm{O}$ with $1 \%$ of $\mathrm{MeOH}$ in a concentration range from $0.01 \mu \mathrm{M}$ to $100 \mu \mathrm{M}$. Treatment was performed on four replicates in the same plate, and experiments were repeated at least three times independently and for each strain. Control was treated with $\mathrm{H}_{2} \mathrm{O} / 1 \%$ $\mathrm{MeOH}$. Beforehand, the effect of adding 1\% methanol compare to the liquid medium without methanol was tested, showing that methanol has no effect on bacterial growth. Growth was monitored by measuring every $30 \mathrm{~min}$ the absorbance of the microcultures at $600 \mathrm{~nm}$ with a microplate reader (UV-Vis SpectraMax 190 Microplate Reader, Molecular Devices) for $24 \mathrm{~h}$ of incubation in the presence or absence of the tested compounds. Results analysis were performed on final absorbance values within experiments. Growth inhibition for each target organism was calculated using the following expression:

$100 \times\left(1-\left(\frac{\Delta O D_{600 \mathrm{~nm}, 0-24 \mathrm{~h}} \text { in } \mathrm{HPO}}{\Delta O D_{600 \mathrm{~nm}, 0-24 \mathrm{~h}} \text { in control }}\right)\right.$ 


\subsection{IMPALA procedure}

The insertion of the molecule into an implicit bilayer was computed by the IMPALA procedure as described previously $[44,45]$. Briefly, this method allows the study of the insertion of a compound in a modelled membrane using simple restraint functions designed to mimic the membrane properties. The IMPALA method allows us to predict if a molecule is able to penetrate a membrane and how deep it inserts into the lipid phase.

The membrane is described as a continuous medium whose properties vary along the axis perpendicular to the bilayer plane ( $\mathrm{z}$ axis). The forcefield is set to mimic a membrane in aqueous environment by considering 1 ) the hydrophobic effect between the membrane and a solute $\left(\mathrm{E}_{\mathrm{pho}}\right)$ and 2 ) the perturbation effect of the solute on the lipid acyl chain organization $\left(\mathrm{E}_{\text {lip }}\right)$. The two restraints are calculated and summed at each position of the 13-HPOT molecule into the implicit membrane; the molecule is systematically moved along the $\mathrm{z}$ axis by $1 \AA$ A steps, from one side of the membrane to the other. A profile of the total energy restraint $\left(E_{\text {env }}=E_{\text {pho }}+E_{\text {lip }}\right)$ as a function of the mass centre position of 13-HPOT into the implicit bilayer is then obtained.

\subsection{Isothermal titration calorimetry}

For isothermal titration calorimetry (ITC), large unilamellar vesicles (LUVs) were made. Lipid films were prepared the same way as for calcein leakage experiments. This time no fluorescent probes were added while hydrating them. The flask was maintained at a temperature above the transition phase temperature for $1 \mathrm{~h}$ and vortexed every $10 \mathrm{~min}$. Thereafter, the suspension underwent 5 freeze-thaw cycles and then extruded 15 times through polycarbonate filters with a pore diameter of $100 \mathrm{~nm}$, in order to get LUVs. The size and homogeneity of the produced liposomes was verified by Dynamic Light Scattering. BPM liposomes had an average diameter $120 \pm 5.7 \mathrm{~nm}$.

The measurements were performed with a VP-ITC from Microcal (Microcal Inc., Northampton, MA, USA) at $26^{\circ} \mathrm{C}$. Before starting, solutions were degassed by stirring under vacuum or by ultrasonication. The sample cell (volume $=1,4565 \mathrm{~mL}$ ) was filled out with $10 \mathrm{mM}$ Tris$\mathrm{HCl}$ buffer at $\mathrm{pH} 7.4$ (blank) or 13-HPOT at $10 \mu \mathrm{M}$ (below CMC) in $10 \mathrm{mM}$ Tris $\mathrm{HCl}$ buffer at $\mathrm{pH} 7.4$ and the reference cell was loaded with milli-Q water. Small aliquots of LUV suspension at $2 \mathrm{mM}$ in Tris $\mathrm{HCl}$ buffer at $\mathrm{pH} 7.4$ were added to the sample cell with a software-controlled syringe (volume $=300 \mu \mathrm{L}$ ). The first injection was $2 \mu \mathrm{L}$ (not taken into account for data treatment) and was followed by 28 injections of $10 \mu \mathrm{L}$. A spacing time of $360 \mathrm{~s}$ was used between each injection to reach a steady state before each new injection. The sample cell was stirred continuously at $305 \mathrm{rpm}$ during experiments. By subtracting the values obtained for the blanks (same experiment in DMSO and without 13-HPOT) from the observed heats, the effective heats were determined. Raw data were treated using ORIGIN7 software (Originlab, Northampton, USA) according to the cumulative model described hitherto [46] and previously applied for other surfactants $[47,48]$. The thermodynamic parameters were calculated as described in [46] and calculations provided the values of : the molar free energy $(\Delta \mathrm{G})$, the molar enthalpy change $(\Delta \mathrm{H})$ and the molar entropy change (T $\Delta S)$ of the binding reactions as well as the binding coefficient $(\mathrm{K})$ related to the affinity of 13-HPOT for LUVs. All experiments were carried out at least three times, each time with freshly prepared LUVs.

\subsection{Calcein leakage}

POPE/POPG/CL (60:21:11) small unilamellar vesicles (SUVs) were prepared as described previously $[41,49,50]$. While hydrating the film, $10 \mathrm{mM}$ calcein in $10 \mathrm{mM}$ TRIS-HCl buffer $\mathrm{pH} 7.4$ was added. The lipid dispersion was maintained at $37^{\circ} \mathrm{C}$ for at least $1 \mathrm{~h}$ and vortexed every $10 \mathrm{~min} .5$ cycles of freeze-thawing were applied to spontaneously form multilamellar vesicles. To obtain SUVs, this suspension was sonicated to clarity ( 5 cycles $\times 2 \mathrm{~min}$ ) using a probe with $400 \mathrm{~W}$ amplitude keeping the suspension in an ice bath. Finally, generated titanium particles were removed from SUV solution by centrifuging during $10 \mathrm{~min}$ at $6200 \mathrm{rpm}$. The unencapsulated calcein was removed from the SUV dispersion by the Sephadex G65 mini-column separation technique [51]. The actual phospholipid content of each preparation was determined by phosphorus assay [52] and the concentration of liposomes was adjusted for each type of experiment to $5 \mu \mathrm{M}$ in $10 \mathrm{mM}$ TRIS-HCl at pH 7.4 buffer.

Fluorescence was measured as previously described in [51] and with a Perkin Elmer (model LS50B) fluorescence spectrometer equipped with polarizers. Total amount of calcein release was determined by adding Triton-X100 (0.2\%) to a liposome suspension that dissolved the lipid membrane without interfering with the fluorescence signals. The emission and excitation wavelengths were set at $517 \mathrm{~nm}$ and $467 \mathrm{~nm}$, respectively. A fluorescence signal of $750 \mu \mathrm{L}$ of SUV was first recorded as a baseline, followed by the addition of 13-HPOT (at $\mathrm{t}=30 \mathrm{sec}$ ) in 7 different concentrations while continuing the recording for $900 \mathrm{~s}$. The amount of calcein released after time $t$ was calculated according to [53]:

$R F(\%)=100 \frac{\left(I_{t}-I_{o}\right)}{\left(I_{\max }-I_{o}\right)}$

where RF is the fraction of calcein released, $I_{o}, I_{t}$ and $I_{\max }$ are the fluorescence intensities measured at the beginning of the experiment, at time $t$ and after the addition of $0,2 \%$ Triton X-100, respectively. All experiments were carried out at least three times, each time with freshly prepared SUVs.

\subsection{Langmuir monolayer}

Adsorption experiments at constant surface area were performed in a Kibron Minitrough $\left(24 \mathrm{~cm}^{3}\right)$ placed on a vibration-isolated table and equipped with a Wilhelmy wire. Experiments in the absence or in the presence of lipids at the interface were made as previously described [50].

The subphase was $10 \mathrm{mM}$ Tris- $\mathrm{HCl}$ buffer at $\mathrm{pH} 7.4$ (prepared with milliQ water) and continuously stirred with a magnetic stirrer. Lipid molecules chloroform/methanol (2/1, v/v) mixture were spread at the air-water interface in order to reach desired initial surface pressure. After 15 min waiting for the solvent evaporation and film stabilization, 13-HPOT dissolved in DMSO was injected underneath the pre-formed lipid monolayer to a final concentration of $12.7 \mu \mathrm{M}$ in the subphase (below the critical micellar concentration of 13-HPOT which is $25.4 \pm 1.9 \mu \mathrm{M}$ (data not shown)) in order to have it in a monomer form. The adsorption of 13-HPOT at the lipid monolayer was followed by the increase of the surface pressure over time as described previously $[49,50]$. As a control, the same volume of pure DMSO was injected underneath the lipid monolayer and no change of the surface pressure was observed. The maximal insertion pressure (MIP), defined as the surface pressure above which 13-HPOT no longer adsorbs into the lipid monolayer, was determined at the intersection of linear regression of the plot $\Delta \pi v s \pi_{\mathrm{i}}$ with the $\mathrm{x}$-axis. The "differential $\pi_{\mathrm{o}}\left(\mathrm{d} \pi_{\mathrm{o}}\right)$ " parameter was calculated as follows:

$d \pi_{0}=\Delta \pi_{0}-\pi_{e}$

where $\Delta \pi_{0}$ corresponds to the y-intercept of the linear regression of the $\Delta \pi v s \pi_{\mathrm{i}}$ plot, and $\pi_{e}$ is the surface pressure increase at the equilibrium obtained in an independent experiment performed at the same 13HPOT concentration but without lipids spread at the interface. The uncertainty in the MIP and in the $\mathrm{d} \pi_{\mathrm{o}}$ were calculated as described previously [54].

\subsection{Hypermatrix docking method}

Hypermatrix is a simple docking method described in $[49,55]$. Like it was recently done for saponins [56] and amphiphilic azobenzenes 


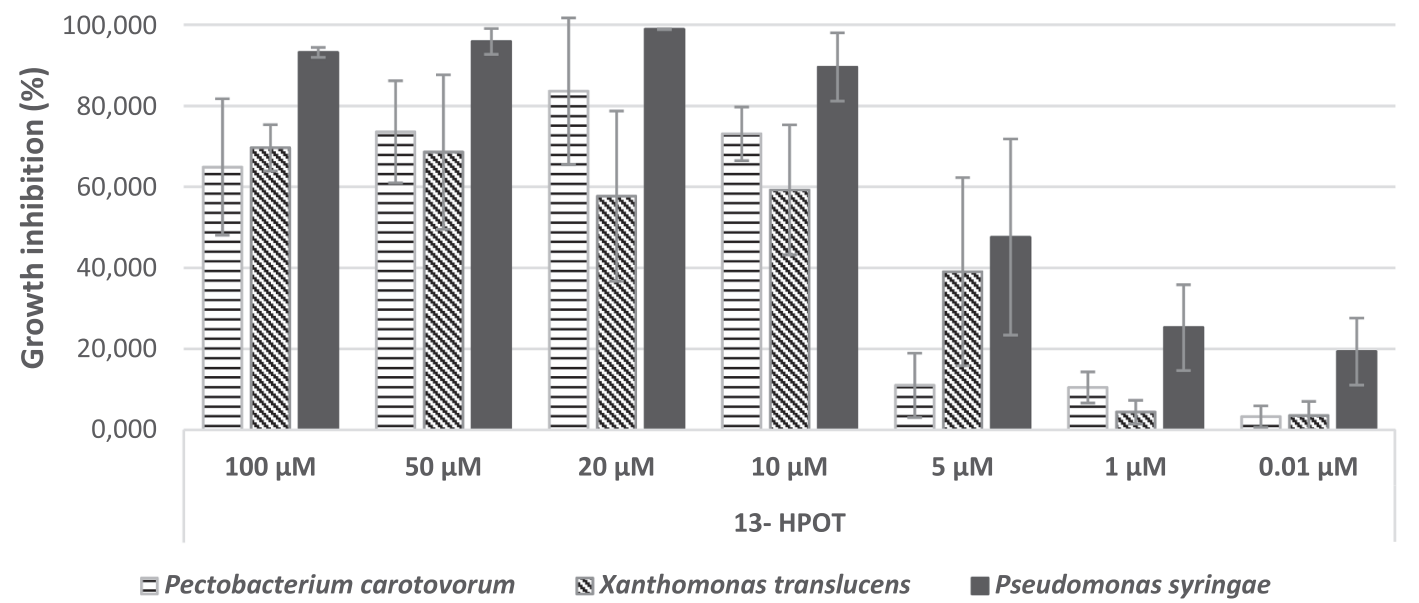

Fig. 1. Effect of 13-HPOT on the growth of three bacteria of agronomic interest. Growth inhibition percentages were calculated based on the optical density measured for each condition. Horizontally hatched bars indicate results for Pectobacterium carotovorum, hatched bars for Xanthomonas translucens and black bars for Pseudomonas syringae pv. syringae. Data represent mean \pm standard deviation of three independent experiments.

[34], it was used to dock 13-HPOT to different lipid molecules. 13HPOT is fixed at a central position of the system and oriented at the hydrophobic/hydrophilic interface and the lipid molecule, also oriented at the lipid/water interface, is positioned around the HPOT molecule by rotations and translations (more than $10^{7}$ positions tested). For each position, an interaction energy is calculated, based on an empirical forcefield [57]. The most stable assembly is the one having the lowest energy value.

\subsection{Big monolayer method}

The Big Monolayer (BM) method allows to visualize lipid domains and was used as described $[38,49,56]$. The first step consists in calculating the paired interactions between molecules, according to Hypermatrix (see above). In this study, these pairs were HPOT/POPE, HPOT/POPG, HPOT/CL, POPE/POPG, POPE/CL and POPG/CL. For each pair, a Boltzmann energy is calculated by adding the interaction energy of each relative position tested multiplied by the probability of the position. After that, a grid of 40,000 $(200 \times 200)$ molecules, randomly positioned at the beginning, is constructed and the energy of the configuration is calculated. Then, random permutations are performed, and the energy of the new configuration is again calculated. A Monte Carlo procedure of 100000 steps permits to minimize the whole system. Three repetitions of each system were calculated. At the end, each molecule is represented by a pixel, generating an image of the molecular domains formed.

\subsection{Laurdan generalized polarization}

For Laurdan generalized polarization experiments, multilamellar vesicles (MLVs) were prepared based on $[38,58]$. POPE, POPG and CL in proportion 60:21:11 were dissolved in a chloroform/methanol mixture $(2 / 1, v / v) .13$-HPOT was added to the lipid mixture to reach a lipid:HPOT molar ratio of $5: 1$. The solvent was evaporated under a gentle stream of nitrogen to obtain a dried lipid film which was maintained under vacuum overnight. The resulting film was hydrated with $10 \mathrm{mM}$ TRIS-HCl buffer at $\mathrm{pH}$ 7.4 prepared from Milli-Q water and $1 \mu \mathrm{L}$ of Laurdan solution prepared in DMSO was added in order to obtain a final concentration of $5 \mathrm{nM}$. The lipid dispersion was maintained at a temperature well above the transition phase temperature of the lipid for at least $1 \mathrm{~h}$ and vortexed every $10 \mathrm{~min}$.

Fluorescence of Laurdan in MLVs was monitored at various temperatures (between 20 and $50{ }^{\circ} \mathrm{C}$ by step of $5{ }^{\circ} \mathrm{C}$ ) with a Perkin Elmer LS50B fluorescence spectrometer. Samples were placed in $10 \mathrm{~mm}$ pathlength quartz cuvettes under continuous stirring while the cuvette holder was thermostated with a circulating bath. Samples were equilibrated at each temperature for 10-15 min before measurement was performed. The excitation wavelength was set to $360 \mathrm{~nm}$ (slit $=2.5 \mathrm{~nm}$ ), and at least 10 measurements of emission intensities at $440 \mathrm{~nm}$ and $490 \mathrm{~nm}$ were recorded and averaged for each sample and the blank (DMSO) at each temperature. An emission spectrum from $400 \mathrm{~nm}$ to $600 \mathrm{~nm}$ (slit $=4,5 \mathrm{~nm}$ ) was also recorded for each sampletemperature combination. Generalized polarization (GP) of Laurdan was then calculated according to $[59,60]$ :

$G P=\frac{I_{440}-I_{490}}{I_{440}+I_{490}}$

where $I_{440}$ and $I_{490}$ are the blank-subtracted emission intensities at $440 \mathrm{~nm}$ and $490 \mathrm{~nm}$, respectively. All experiments were carried out at least three times, each time with freshly prepared MLVs.

\section{Results and discussion}

\subsection{Evaluation of 13-HPOT inhibitory activity against plant bacterial pathogens}

The first objective of this study was to evaluate the antibacterial effect of 13-HPOT, in a range of defined concentrations, against three crop pathogenic bacteria: $P$. carotovorum, $P$. syringae and $X$. translucens. Bacteria were grown in vitro in 96-well plates in liquid medium in presence or absence (control) of 13-HPOT for $24 \mathrm{~h}$. After subtracting growth values from the sterile medium in the presence of HPOT (different concentration tested) and comparing these values to the positive control (bacterial medium + solvent), growth inhibition percentages were calculated for each tested concentration and each pathogen (Fig. 1).

Fig. 1 shows that 13-HPOT induced a dose-dependent antibacterial effect on the three pathogens. The molecule seems less effective below $10 \mu \mathrm{M}$, whereas at concentrations above $10 \mu \mathrm{M}$ the inhibition percentages are increasing up to $100 \%$ in the case of $P$. syringae. For all the concentrations tested, 13-HPOT seems to be more active and at lower concentrations on hemibiotrophic $P$. syringae than necrotrophic on $P$. carotovorum and on $X$. translucens. Clearly, 13-HPOT exhibits in vitro antimicrobial activities against these Gram-negative bacteria. As results are promising with $P$. syringae, the rest of the study is focused on this specific case. 


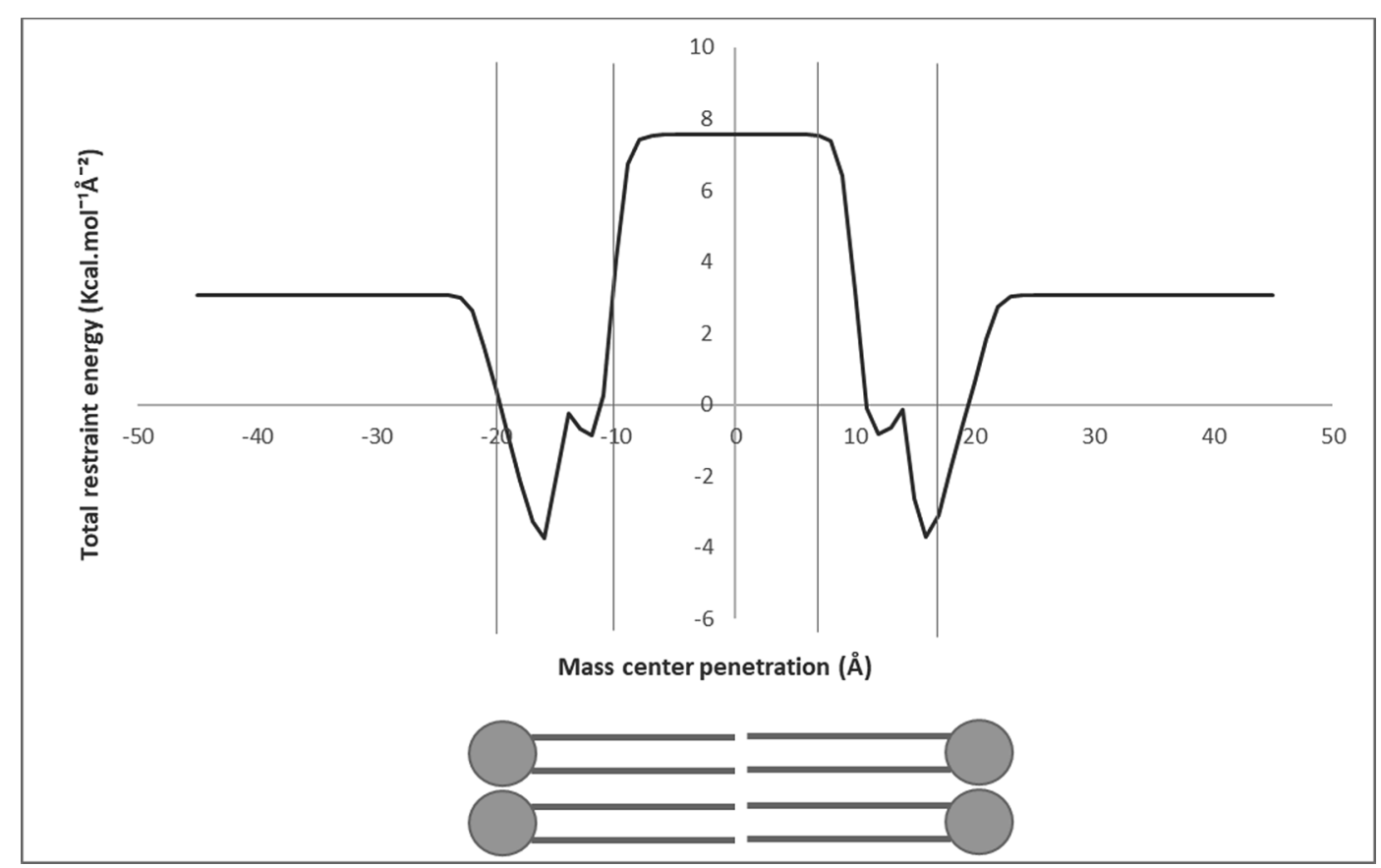

Fig. 2. Total restraint energy as a function of 13-HPOT mass centre penetration in an implicit bilayer, represented below, calculated by the IMPALA procedure. The vertical lines correspond (from left to right) to the interface between the bilayer and the aqueous phase ( $\mathrm{z}=20 \AA$ ), the interface between the hydrocarbon core and the lipid hydrophilic head $(\mathrm{z}=14 \AA)$, and the centre of the bilayer ( $\mathrm{z}=0 \AA$ ).

\subsection{Biophysical investigation of the antibacterial mechanism}

\subsubsection{Investigation of 13-HPOT interactions with bacterial IM-mimicking models}

Since the membrane could be one target of antibacterial molecules, we have investigated, in silico, the interaction of 13-HPOT with the BPM. In a first approach, the IMPALA procedure [44] was applied to predict the ability of 13-HPOT to insert into an implicit model membrane. The molecule was systematically moved along the $\mathrm{z}$ axis from one side of the membrane to the other and the total restraint $\left(E_{\text {pho }}+E_{\text {lip }}\right)$ was calculated for each position. A profile of the energy restraints as a function of the mass centre penetration into the implicit bilayer was then obtained (Fig. 2).

A sharp drop of energy from the aqueous phase to the membrane surface is observed (Fig. 2), indicating that the entry of the molecule into membrane is clearly favourable. However, the large positive value of the energy in the hydrophobic region of the membrane suggests that the crossing of the membrane by 13-HPOT is unlikely. The most favourable positions corresponding to the lowest value of restraint energy are those located at the level of the lipid polar head/lipid hydrocarbon chain interface at around $14 \AA$. Therefore, 13-HPOT is predicted to be able to interact with a membrane and to fit into but could not cross the bilayer and would remain localized at the outer leaflet.

In a second step, we experimentally analysed, in vitro, the thermodynamic aspect of the interaction between 13-HPOT and bacterial mimicking model membranes using the ITC technique. LUVs were formed with a lipid composition mimicking Pseudomonas sp. IM; palmitoyl oleyl phosphatidylethanolamine (POPE), palmitoyl oleyl phosphatidylglycerol (POPG) and cardiolipin (CL) as model phospholipids, in a molar ratio 60:21:11 respectively $[26,37,61]$. The raw binding profile shows weak positive peaks that decrease over time (Fig. 3A) and become negative (corresponding to the heat flow of the drop fall) with the successive liposome injections.

After subtraction of the heat of dilution from the blank titration of the liposomes into the buffer, the thermodynamic parameters were calculated (Table 1). The binding reaction to liposomes mimicking bacterial IM was spontaneous $(\Delta \mathrm{G}<0)$, endothermic $(\Delta \mathrm{H}>0)$ and generated a positive change of entropy $(\Delta S>0)$. Data demonstrate that 13-HPOT can bind to these liposomes, suggesting an interaction with the lipid phase of the IM. The binding coefficient (K) is quite high compared to other surfactant molecules $[46,48]$, suggesting that 13HPOT presents a high affinity for IM membrane lipids. As the absolute values of entropy change is higher than the absolute value of enthalpy change, the binding is notably driven by hydrophobic interactions [62].

Altogether, both in silico and in vitro results indicate that 13-HPOT is attracted by and interact with IM-mimicking membranes and that the hydrophobic interactions are the main driving forces for the interactions with bacterial phospholipids.

3.2.2. Investigation of the mechanism : A non-detergent-like mode of action Since most amphipathic antimicrobial agents derive their effectiveness from a detergent-like action [63], we have investigated the ability of 13-HPOT to permeabilize model membranes by using calcein leakage assays. Following the interaction between 13-HPOT and the biomimetic membrane, calcein can be released from the internal aqueous medium of the vesicles, where it is self-quenched, into the external aqueous environment, if the structure of the membrane is affected by the interaction.

No permeabilization effect was observed (values less than 10\%) regardless of the tested concentrations of 13-HPOT in a range from 0.1 to $12.5 \mu \mathrm{M}$ (data not shown). The same result was observed with plant membrane models (unpublished data) suggesting that 13-HPOT would not derive its mode of action from a mechanism of solubilization of the membrane but rather from a more subtle modification of the membrane organization that could lead to the activation of a signalling cascade and de facto to cell death. Actually, some antibiotics can also exert their biological activity against microbes by interacting directly and specifically with the membrane lipid parts $[23,28]$. Discovered a few years ago in Bacillus subtilis [64] and more recently in other bacteria $[26,27,65]$, functional domains might be the target for novel antibacterial agents. Modifications of these domains might be lethal as they are related to important cell processes (cell division, signal transduction, biofilm formation, etc.) $[64,65]$. 

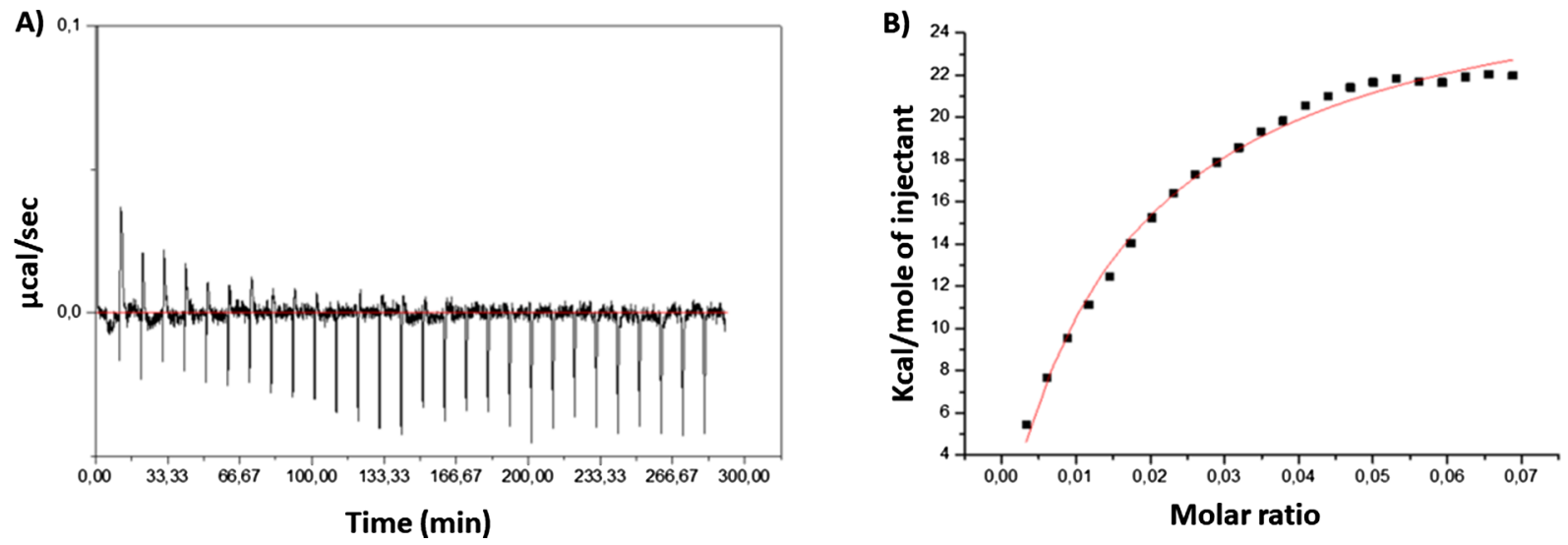

Fig. 3. Thermodynamic profiles of one ITC experiment conducted with 13-HPOT and the bacterial liposome composition. A) Raw thermogram (heat flow) observed upon addition of POPE/POPG/CL vesicles (2 mM) into $10 \mu \mathrm{M}$ 13-HPOT, both in buffer (10 mM Tris-HCl, pH 7.4). B) Cumulative heats of reaction as obtained from the integration of the thermogram shown in A. Red curve correspond to the fitting from which the thermodynamic parameters are calculated.

Table 1

Summary table of thermodynamic parameters calculated based on the cumulative heats of reactions.

\begin{tabular}{lll}
\hline & Mean values & Standard deviations \\
\hline$\Delta \mathrm{H}(\mathrm{J} / \mathrm{mol})$ & 1,10 & 0,40 \\
$\mathrm{~K}\left(\mathrm{mM}^{-1}\right)$ & 49,78 & 7,38 \\
$\Delta \mathrm{G}(\mathrm{J} / \mathrm{mol})$ & $-36,83$ & 0,37 \\
$\mathrm{~T} \Delta \mathrm{S}(\mathrm{J} / \mathrm{mol})$ & 37,93 & 0,65 \\
\hline
\end{tabular}

\subsubsection{Involvement of specific lipids in BPM - 13-HPOT interactions}

To better understand a potential specific interaction between 13HPOT and bacterial lipids, adsorption experiments into pure lipid monolayers composed of POPE, POPG or CL were performed using the Langmuir monolayer technique. Indeed, monolayers are excellent alternatives to study interactions in two-dimensional arrangements of amphiphilic molecules and well suited for mimicking membrane surface processes $[66,67]$. The adsorption profile was monitored by the increase of the surface pressure over time at a constant trough area. From the plot of the maximal surface pressure variation $(\Delta \Pi)$ versus the initial surface pressure $\left(\Pi_{\mathrm{i}}\right)$ (data not shown), two different parameters have been calculated: the MIP which reflects the penetration power of the molecule into a lipid monolayer and corresponds to the pressure above which a surface-active molecule is no longer able to insert into a lipid monolayer, and the differential $\Pi_{\mathrm{o}}\left(\mathrm{d} \Pi_{\mathrm{o}}\right)$ which indicates the (non)attracting effect of the lipid towards the molecule.

The $\Delta \Pi v s$. Пi curves (data not shown) decreased linearly with increasing values of $\Pi_{i}$, suggesting that a higher packing of lipid molecules at the air-water interface leads to a weaker adsorption of 13-HPOT into the monolayer, as observed for various amphiphilic molecules $[24,49,50]$. All the MIP values are higher than the lateral pressure supposed to prevail within biological membranes (30-35 mN/m) [68] (Fig. 4), suggesting again that 13-HPOT could insert into natural plasma membranes. These results agree with the IMPALA results but should be interpreted with caution because monolayers studied here are very far from representing the IM. The positive values of $\mathrm{d} \Pi_{0}$ confirm the affinity of 13-HPOT for the individual lipid monolayers. The affinity was significantly higher for monolayers composed of PE than PG or CL.

In silico docking between 13-HPOT and the three lipids tested experimentally was then performed to obtain a description of the interaction at the atomic level. For all the lipids, the total energy of interaction was negative, confirming an energetically favourable interaction between 13-HPOT and the lipids mimicking the BPM (Fig. 5). As experimentally, 13-HPOT presents less affinity for CL, with even a positive hydrophilic component. The absolute value of interaction energy is significantly higher in presence of $\mathrm{PE}$, confirming a specific interaction

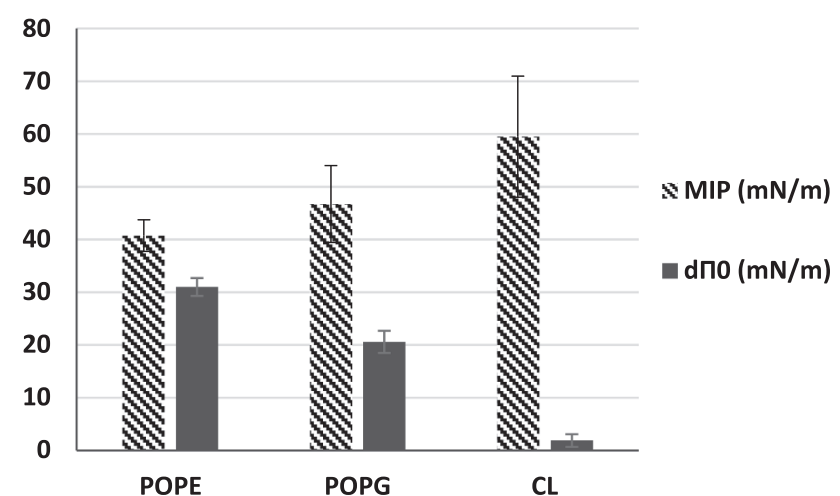

Fig. 4. Adsorption of 13-HPOT into lipid monolayers: POPE: palmitoyl oleyl phosphatidylethanolamine, POPG: palmitoyl oleyl phosphatidylglycerol, CL: cardiolipin. Maximal insertion pressure (MIP) in hatched bars and differential П० $\left(\mathrm{d} \Pi_{0}\right)$ value in black bars.

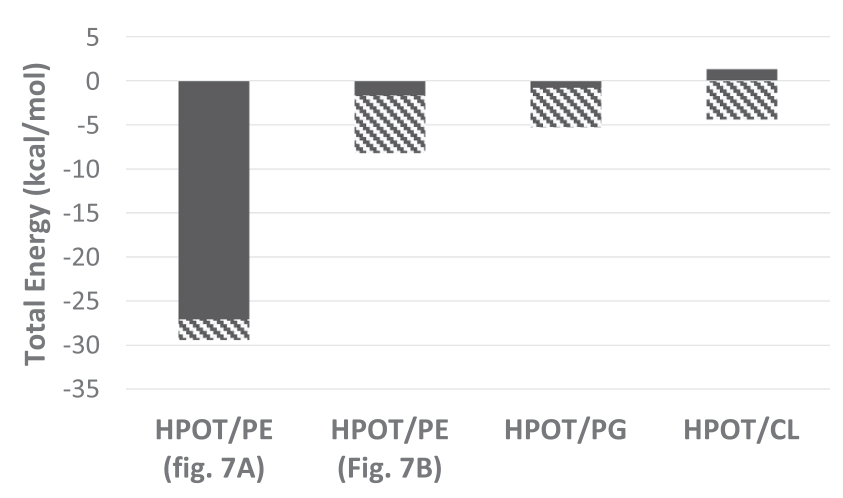

- Hydrophilic energy Hydrophobic energy

Fig. 5. Total energy of interaction of 13-HPOT with POPE (palmitoyl oleyl phosphatidylethanolamine), POPG (palmitoyl oleyl phosphatidylglycerol), and CL (cardiolipin) in a multimolecular monolayer assembly, calculated by the Hypermatrix docking method. In black: hydrophilic component of the energy; hatched: hydrophobic component of the energy.

with this phospholipid. Moreover, we observe two possible interaction cases for PE. As shown on Fig. 6A (upper panel), the $\mathrm{OOH}$ group and part of the alkyl chain of 13-HPOT interact respectively with the ester group and the beginning of the alkyl chain of the lipid. The other energetically favourable interaction is between the amine group of $\mathrm{PE}$ and the carboxy group of 13-HPOT (Fig. 6B, upper panel), increasing significantly the polar interactions but decreasing the hydrophobic energy 


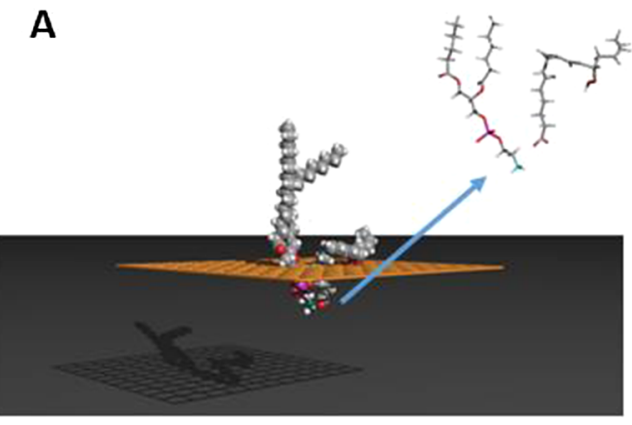

C

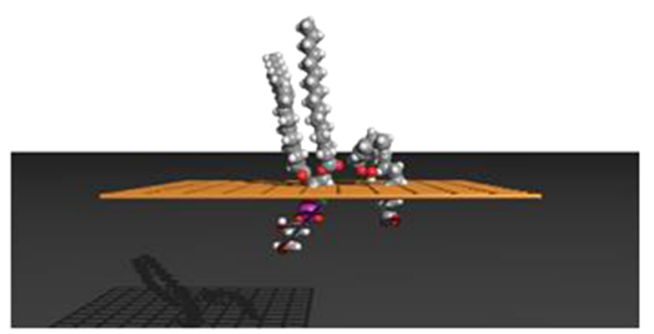

B

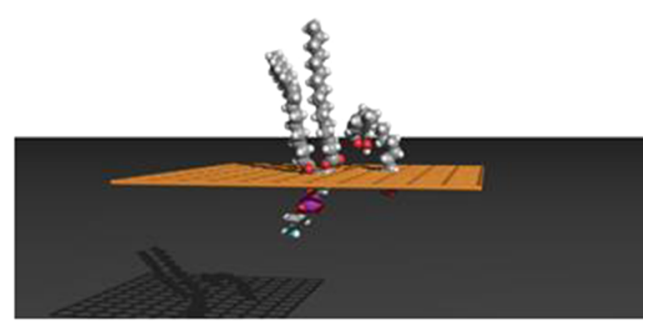

D

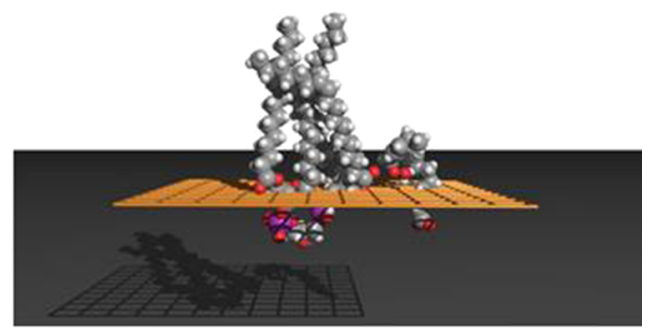

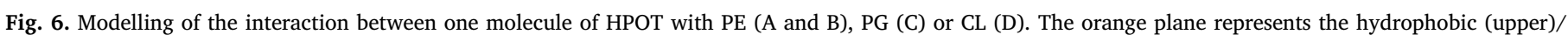
hydrophilic (Below) interface. (For interpretation of the references to colour in this figure legend, the reader is referred to the web version of this article.)

(Fig. 5). For the two other lipids, the interaction resembles the first one of PE/13-HPOT (Fig. 6C-D). When comparing the energy of interaction (from Fig. 5), PE is ahead of PG, which is also ahead of CL.

Data from Langmuir monolayer experiments and docking calculations strongly suggest that PE plays a key role in the interaction between 13-HPOT and the BPM. Then, one can wonder how these specific interactions could be related to the biological effects of 13-HPOT. It is well known that such interactions can influence the membrane organization, the distribution and localization of certain proteins leading notably to the activation of signalling cascade reactions $[26,65,69]$.

\subsubsection{Effect of 13-HPOT on the BPM structure and organization}

Our final step was to investigate the effect of 13-HPOT on the BPM order and organization. First, to determine the potential effect of lipid organization on the interaction with 13-HPOT, measurements made on a monolayer of the POPE/POPG/CL ternary mixtures were performed (Fig. 7). The corresponding parameters are $23.3 \pm 3.9 \mathrm{mN} / \mathrm{m}$ for the MIP and $1.9 \pm 0.9 \mathrm{mN} / \mathrm{m}$ for the $\mathrm{d} \Pi 0$. In this case, the MIP value was

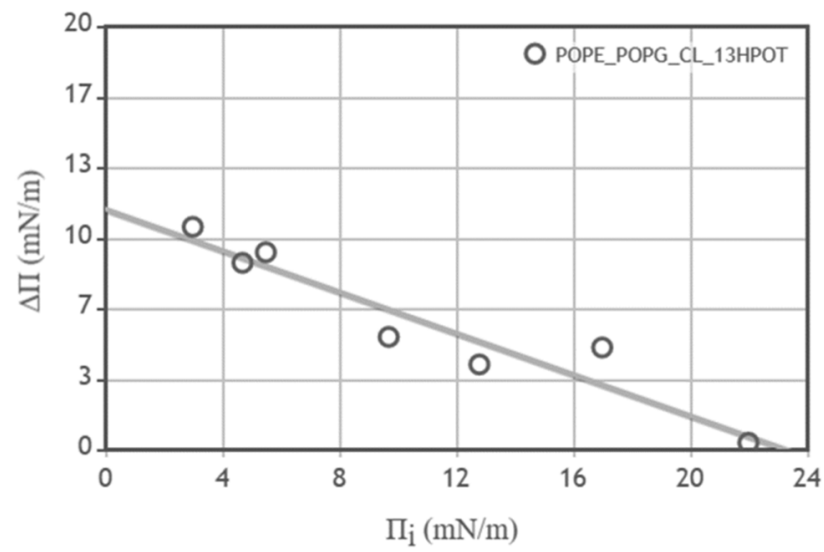

Fig. 7. Adsorption of 13-HPOT into a POPE/POPG/CL (60/21/11, molar ratio) monolayer. Plot of the maximal surface pressure variation $(\Delta \Pi)$ versus the initial surface pressure (Пi) for the monolayer. lower although remaining not too far from the lateral pressure within biological membranes $(30-35 \mathrm{mN} / \mathrm{m})$. In addition, the value of $\mathrm{d} \Pi 0$ was significantly reduced, similar to that obtained with the monolayers CL. This suggests that, in the ternary mixture (nigh to real BPM), the lipid organization would disturb but not hinder the interaction between 13-HPOT and the different lipids. The organization can be such that the POPE and POPG molecules are inaccessible or misdirected to interact with 13-HPOT.

In a second time, the simulation of the lateral distribution of 13HPOT within the ternary lipid mixture was obtained using the BM approach (Fig. 8). The presence of 13-HPOT clearly influences the lateral organization of bacterial lipids. By comparing the two simulations, it is predicted that the presence of HPOT modifies the lipid domains (Fig. 8). This in silico simulation suggests that CL is excluded from the lipid bulk when 13-HPOT is present and forms large CL clusters. Formation of CL domains have been reported in the membranes of Escherichia coli and $B$. subtilis [26]. These domains are localized in the polar and septal regions, but it is still not clear how these domains form. CL domains are known to induce a curvature in the plasma membrane at the poles and to be involved in cell division process $[26,64]$. The modification of the membrane organization and of the distribution of CL induced by the presence of 13-HPOT might influence the curvature and fluidity of the membrane which could impact the cell division process or even cause cell death.

To experimentally investigate the effect of 13-HPOT on the membrane order, we evaluated the lipid bilayer fluidity by the Laurdan polarization. Laurdan is a fluorescent probe that readily partitions at the hydrophilic/hydrophobic interface of bilayers [70]. According to its environment, the fluorescence properties of Laurdan change. When inserted into a lipid bilayer in the gel state, the fluorescence is maximal at an emission wavelength around $440 \mathrm{~nm}$. As soon as the bilayer undergoes a liquid phase transition, the maximum fluorescence intensity is shifted toward higher emission wavelengths. This phenomenon is socalled the "red-shift" and is awarded to an increased presence and mobility of water molecules around the probe, directly linked to the lipid bilayer order [60]. From raw data, Laurdan Generalized Polarization (GP) parameter was calculated to monitor the change in lipid order. In our experiments, Laurdan GP was used to determine the effect 

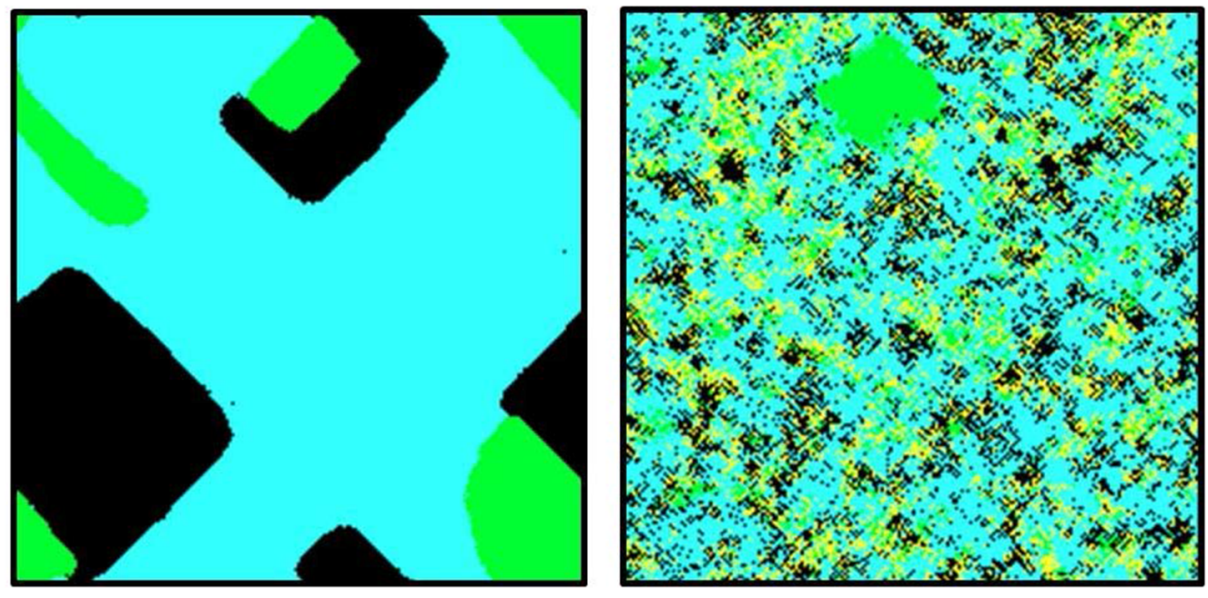

Fig. 8. Monolayer simulations of $\mathrm{PE} / \mathrm{PG} / \mathrm{CL} / \mathrm{HPOT}$ system (56/19/10/16 M ratio) obtained by the Big Monolayer method in a grid of $200 \times 200$ molecules. Each molecule is represented by a pixel: blue: PE; black: PG green: CL and yellow: HPOT. Left picture represents the simulations without 13HPOT. Right picture represents one of the simulations in presence of 13-HPOT. (For interpretation of the references to colour in this figure legend, the reader is referred to the web version of this article.)

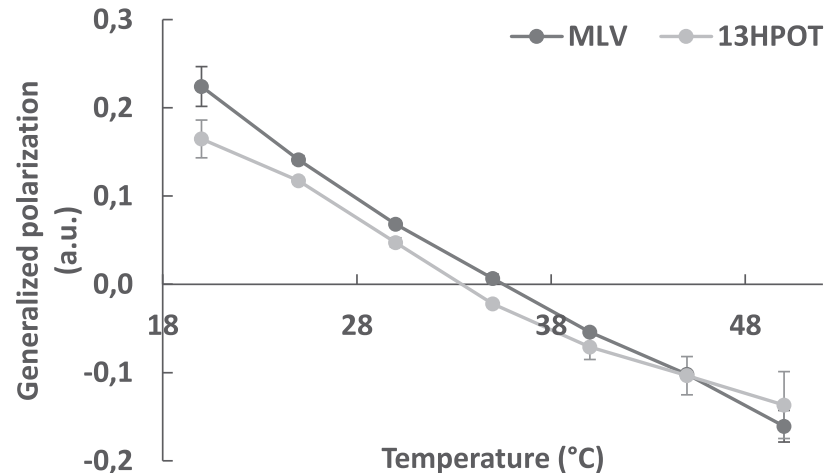

Fig. 9. Evolution of Laurdan generalized polarization as a function of temperature for POPE/POPG/CL MLVs $(50 \mu \mathrm{M})$ in the absence or presence (lipid:HPOT molar ratio 5:1) of 13-HPOT. Dark grey: MLVs only; Grey: MLVs + 13-HPOT.

of 13-HPOT on the lipid order at the hydrophilic/hydrophobic interface at different temperatures.

Fig. 9 shows the temperature dependence of Laurdan GP for POPE/ POPG/CL MLVs mixed with 13HPOT (lipid:HPOT ratio of 5:1). Comparatively to other bioactive molecules (neamine derivatives, sorgoleone, etc.), the GP values are moderated [37,71]. In presence of 13HPOT, the Laurdan GP values are slightly decreased for temperature below $40-45{ }^{\circ} \mathrm{C}$, whereas above $40-45{ }^{\circ} \mathrm{C}$, values are slightly higher than those of pure MLVs. Hence, for temperatures below $40{ }^{\circ} \mathrm{C}, 13-$ HPOT tends to fluidify, i.e. disorganize the bilayer. At higher temperatures, it has rather a slight ordering effect on the bilayer. Interestingly, 13-HPOT behaviour seems to be related to the temperature, which might be explained by fluctuations of the lipid phase. Further investigations are needed to better understand this specific behaviour.

\section{General discussion and conclusion}

Amphipathic molecules are good candidates for an optimal interaction with lipid membranes. This is especially critical for antibacterial agents active against Gram-positive or -negative bacteria when unable to pass through porins [28]. Fatty acid hydroperoxides appear hence as potential biocidal candidates. Following previous studies [17,18,24], 13-HPOT is the most promising.

In this study, antibacterial assays showed that 13-HPOT exhibits a high antibacterial activity against the three Gram-negative bacteria tested: $P$. carotovorum, $P$. syringae and $X$. translucens. We have also demonstrated that 13-HPOT interacts with lipid bilayers mimicking the Pseudomonas IM. However, it cannot cross the bilayer and rather remains localized at the outer leaflet, likely due to its two polar groups forcing it to stay into the region close to the membrane interface. 13HPOT does not permeabilize the IM models, unlike other small amphiphile molecules that have a detergent-like mechanism of action. Its mode of action should involve either more subtle changes of membrane properties, such as its lateral organization and distribution, and/or an interaction with proteins within the membrane. Indeed, 13-HPOT showed energetically favourable interactions with all the tested lipids. The IM of bacteria could thus be the target of our compound. However, one cannot rule out that HPOT could also interact with the outer leaflet of Gram-negative bacteria. The OM lipid composition greatly differs from that of IM, containing mainly LPS and traces of glycerolipids [31]. Preliminary results from our group showed also the ability of 13-HPOT to interact with LPS monolayers (data not shown) but in a way very different from the one observed with the IM lipids.

The interaction of 13-HPOT with PE is the most favourable. The fact that 13-HPOT does not penetrate deeply into the bilayer should render it an inducer of positive spontaneous curvature and, in turn, favours its packing with a negative spontaneous curvature lipid such as PE. It would probably improve packing and limit disordering compared to a more positive-curvature lipid, which is in accordance with the absence of leakage observed in our permeabilization assays [72,73]. This specific and strong lipid interaction can lead to significant changes in the organization of the membrane, and in the distribution and functioning of some membrane proteins [74]. By the way, our Big Monolayer results suggest that 13-HPOT could influence the lateral organization of Pseudomonas lipids. We assume that CL molecules are excluded from the PE/PG areas in the presence of 13-HPOT, with formation of CL clusters. $\mathrm{CL}$ is one important physiological lipid that is organized into microdomains containing proteins that are involved in essential processes $[32,75]$. Therefrom, compounds that influence CL distribution are of great interest in fighting against bacterial resistance to conventional antibiotics in Humans. El Khoury et al. have notably shown that this modification of CL domains causes bacterial cells to have serious morphological defects in their membranes, including an increase in curvature and a decrease in thickness [35]. Using Laurdan polarization measurements, we have shown that 13-HPOT acts on the physical state of the bacterial membrane by slightly fluidizing it. It would be worth to study the influence of these rearrangements on the structure and function of the plasma membrane, particularly using lipids labelled with fluorescent probes. Those lipid organization modifications could influence the conformation and/or function of membrane proteins, notably at the level of the lipid domains present in the bacterial IM. However, it has also been recently demonstrated that lipid domains can be formed in the OM, which may have properties and functions different from what is observed in the IM [69]. Additional studies are needed to further explore this hypothesis.

The biocide activities of 13-HPOT against three bacteria of agronomical interest described in this study are attractive as they could offer 
a track for a new class of biopesticides. In this context, the antibacterial effects of HPOs should be explored on other strains and in planta in a future investigation. Their effects on the outer membrane should also be further studied to have a more global view on the biological activities of HPOs on the bacterial membranes. This is even more true since the composition and structure of LPS differ greatly from one organism to another and from one strain to another [76-78]. The track of the antifungal activity should also be explored in more depth. In the case of plant-pathogen interactions, disrupting or modifying lipid domain organization might be an effective approach to new biopesticides.

\section{Declaration of Competing Interest}

The authors declare that they have no known competing financial interests or personal relationships that could have appeared to influence the work reported in this paper.

\section{Acknowledgements}

E.D. is supported by a « Fonds pour la formation à la Recherche dans l'Industrie et dans l'Agriculture " (FRIA) grant (5100617F) from the FRS-FNRS (Fonds National de la Recherche Scientifique, Belgium). M.D., M. O. and L.L. thank the FRS-FNRS for their position as Senior Research Associates and for grant CDR (J.0014.08 and J.0086.18 projects).

\section{Appendix A. Supplementary material}

Supplementary data to this article can be found online at https:// doi.org/10.1016/j.bioorg.2020.103877.

\section{References}

[1] C. Wasternack, I. Feussner, The oxylipin pathways: biochemistry and function, Annu. Rev. Plant Biol. 69 (2018) 363-386.

[2] E. Blée, Phytooxylipins and plant defense, Prog. lipid Reseach 37 (1998) 33-72.

[3] M. Genva, et al., New insights into the biosynthesis of esterified oxylipins and their involvement in plant defense and developmental mechanisms, Phytochem. Rev. 8 (2019) 343-359.

[4] C. Wasternack, M. Strnad, Jasmonates: News on Occurrence, Biosynthesis, Metabolism and Action of an Ancient Group of Signaling Compounds, Int. J. Mol. Sci. 19 (2018) 2539.

[5] E. Blée, Impact of phyto-oxylipins in plant defense, Trends Plant Sci. 7 (2002) 315-321.

[6] A.J. Koo, Metabolism of the plant hormone jasmonate: a sentinel for tissue damage and master regulator of stress response, Phytochem. Rev. 17 (2018) 51-80.

[7] G. Griffiths, Biosynthesis and analysis of plant oxylipins, Free Radic. Res. 49 (2015) 565-582.

[8] G.A. Howe, Plant hormones: Metabolic end run to jasmonate, Nat. Chem. Biol. 14 (2018) 109-110.

[9] T.V. Savchenko, O.M. Zastrijnaja, V.V. Klimov, Oxylipins and plant abiotic stress resistance, Biochem. 79 (2014) 362-375.

[10] M.L. Fauconnier, M. Marlier, Revue bibliographique: Les lipoxygenases du soja, Biotechnol., Agron. Soc. Environ. 1 (1997) 125-141.

[11] H. Porta, M. Rocha-Sosa, Plant Lipoxygenases. Physiological and Molecular Features, Plant Physiol. 130 (2002) 15-21.

[12] A. Mosblech, I. Feussner, I. Heilmann, Oxylipins: Structurally diverse metabolites from fatty acid oxidation, Plant Physiol. Biochem. 47 (2009) 511-517.

[13] A. Andreou, I. Feussner, Lipoxygenases - Structure and reaction mechanism, Phytochemistry 70 (2009) 1504-1510.

[14] G.A. Howe, A.L. Schilmiller, Oxylipin metabolism in response to stress, Curr. Opin. Plant Biol. 5 (2002) 230-236.

[15] M.L. Fauconnier, R. Welti, E. Blée, M. Marlier, Lipid and oxylipin profiles during aging and sprout development in potato tubers (Solanum tuberosum L.), Biochim. Biophys. Acta - Mol. Cell Biol. Lipids 1633 (2003) 118-126.

[16] M.E. Ghanem, et al., Organ-dependent oxylipin signature in leaves and roots of salinized tomato plants (Solanum lycopersicum), J. Plant Physiol. 169 (2012) 1090-1101.

[17] I. Prost, et al., Evaluation of the Antimicrobial Activities of Plant Oxylipins Supports Their Involvement in Defense against Pathogens, Plant Physiol. 139 (2005) 1902-1913.

[18] G. Granér, M. Hamberg, J. Meijer, Screening of oxylipins for control of oilseed rape (Brassica napus) fungal pathogens, Phytochemistry 63 (2003) 89-95.

[19] B. Zieniuk, A. Fabiszewska, Yarrowia lipolytica : a beneficious yeast in biotechnology as a rare opportunistic fungal pathogen : a minireview, World J.
Microbiol. Biotechnol. 35 (2019) 1-8.

[20] Tran Thanh, H. et al. Toxicity of fatty acid hydroperoxides towards Yarrowia lipolytica: Implication of their membrane fluidizing action. Biochim. Biophys. Acta Biomembr. 1768, 2256-2262 (2007).

[21] E. Deboever, M. Deleu, L. Lins, Plant - Pathogen Interactions : Underestimated Roles of Phyto-oxylipins, Trends Plant Sci. (2019), https://doi.org/10.1016/j. tplants.2019.09.009.

[22] M. Newman, et al., Priming, induction and modulation of plant defence responses by bacterial lipopolysaccharides, J. Endotoxin Res. 13 (2007) 69-84.

[23] G. Sautrey, et al., Negatively charged lipids as a potential target for new amphiphilic aminoglycoside antibiotics: A biophysical study, J. Biol. Chem. 291 (2016) 13864-13874.

[24] M. Deleu, et al., Linoleic and linolenic acid hydroperoxides interact differentially with biomimetic plant membranes in a lipid specific manner, Colloids Surf. B Biointerf. (2018), https://doi.org/10.1016/j.colsurfb.2018.12.014.

[25] A. Jermy, Bacterial physiology: Bacterial lipid rafts discovered, Nat. Rev. Microbiol. 8 (2010) 2455.

[26] I. Barák, K. Muchová, The Role of Lipid Domains in Bacterial Cell Processes, Int. J. Mol. Sci. 14 (2013) 4050-4065.

[27] D. Lopez, G. Koch, Exploring functional membrane microdomains in bacteria : an overview, Curr. Opin. Microbiol. 49 (2017) 76-84.

[28] M.P. Mingeot-Leclercq, J.L. Décout, Bacterial lipid membranes as promising targets to fight antimicrobial resistance, molecular foundations and illustration through the renewal of aminoglycoside antibiotics and emergence of amphiphilic aminoglycosides, Med. Chem. Commun. 7 (2016) 586-611.

[29] M. Aktas, F. Narberhaus, Unconventional membrane lipid biosynthesis in Xanthomonas campestris, Environ. Microbiol. 17 (2015) 3116-3124.

[30] C. Sohlenkamp, O. Geiger, Bacterial membrane lipids: Diversity in structures and pathways, FEMS Microbiol. Rev. 40 (2015) 133-159.

[31] J.C. Henderson, et al., The Power of Asymmetry : Architecture and Assembly of the Gram-Negative Outer Membrane Lipid Bilayer, Annu. Rev. Microbiol. 70 (2016) 255-278.

[32] T. Lin, D.B. Weibel, Organization and function of anionic phospholipids in bacteria, Appl. Microbiol. Biotechnol. 100 (2016) 4255-4267.

[33] I.M. López-lara, O. Geiger, Bacterial lipid diversity, BBA - Mol. Cell Biol. Lipids 1862 (2017) 1287-1299.

[34] A. Franche, et al., Amphiphilic azobenzenes : Antibacterial activities and biophysical investigation of their interaction with bacterial membrane lipids, Bioorg. Chem. 103399 (2019), https://doi.org/10.1016/j.bioorg.2019.103399.

[35] M. El Khoury, et al., Targeting Bacterial Cardiolipin Enriched Microdomains : An Antimicrobial Strategy Used by Amphiphilic Aminoglycoside Antibiotics, Sci. Rep. 1-12 (2017), https://doi.org/10.1038/s41598-017-10543-3.

[36] M. Eeman, et al., Penetration of surfactin into phospholipid monolayers: Nanoscale interfacial organization, Langmuir 22 (2006) 11337-11345.

[37] G. Sautrey, et al., New amphiphilic neamine derivatives active against resistant Pseudomonas aeruginosa and their interactions with lipopolysaccharides, Antimicrob. Agents Chemother. 58 (2014) 4420-4430.

[38] M. Deleu, et al., Effects of surfactin on membrane models displaying lipid phase separation, Biochim. Biophys. Acta - Biomembr. 1828 (2013) 801-815.

[39] H. Tsuchiya, Membrane Interactions of Phytochemicals as Their Molecular Mechanism Applicable to the Discovery of Drug Leads from Plants, Molecules 20 (2015) 18923-18966.

[40] S. Selvaraj, S. Krishnaswamy, V. Devashya, S. Sethuraman, U.M. Krishnan, Progress in Lipid Research Influence of membrane lipid composition on flavonoid - membrane interactions : Implications on their biological activity, Prog. Lipid Res. 58 (2015) 1-13.

[41] M. Ouberai, et al., The Pseudomonas aeruginosa membranes: A target for a new amphiphilic aminoglycoside derivative? Biochim. Biophys. Acta - Biomembr. 1808 (2011) 1716-1727.

[42] R.F. Epand, P.B. Savage, R.M. Epand, Bacterial lipid composition and the antimicrobial efficacy of cationic steroid compounds (Ceragenins), Biochim. Biophys. Acta - Biomembr. 1768 (2007) 2500-2509.

[43] M.L. Fauconnier, M. Marlier, An efficient procedure for the production of fatty acid hydroperoxides from hydrolyzed flax seed oil and soybean lipoxygenase, Biotechnol. Tech. 10 (1996) 839-844.

[44] P. Ducarme, M. Rahman, R. Brasseur, IMPALA: A simple restraint field to simulate the biological membrane in molecular structure studies, Proteins Struct. Funct. Genet. 30 (1998) 357-371.

[45] L. Lins, B. Charloteaux, A. Thomas, R. Brasseur, Computational study of lipid-destabilizing protein fragments: Towards a comprehensive view of tilted peptides, Proteins Struct. Funct. Genet. 44 (2001) 435-447.

[46] H. Heerklotz, J. Seelig, Titration calorimetry of surfactant-membrane partitioning and membrane solubilization, Biochim. Biophys. Acta - Biomembr. 1508 (2000) 69-85.

[47] H. Razafindralambo, S. Dufour, M. Paquot, M. Deleu, Thermodynamic studies of the binding interactions of surfactin analogues to lipid vesicles, J. Therm. Anal. Calorim. 95 (2009) 817-821.

[48] F.N. Zakanda, et al., Interaction of hexadecylbetainate chloride with biological relevant lipids, Langmuir 28 (2012) 3524-3533.

[49] M. Deleu, J.M. Crowet, M.N. Nasir, L. Lins, Complementary biophysical tools to investigate lipid specificity in the interaction between bioactive molecules and the plasma membrane: A review, Biochim. Biophys. Acta - Biomembr. 1838 (2014) 3171-3190.

[50] M.N. Nasir, et al., Interactions of sugar-based bolaamphiphiles with biomimetic systems of plasma membranes, Biochimie 130 (2016) 23-32.

[51] F.N. Fu, B.R. Singh, Calcein permeability of liposomes mediated by type a 
botulinum neurotoxin and its light and heavy chains, J. Protein Chem. 18 (1999) 701-707.

[52] G.R. Bartlett, Calorimetric Assay Phosphorylated for Free Glyceric Acids, J. Biol. Chem. 234 (1958) 469-471.

[53] T. Shimanouchi, H. Ishii, N. Yoshimoto, H. Umakoshi, R. Kuboi, Calcein permeation across phosphatidylcholine bilayer membrane: Effects of membrane fluidity, liposome size, and immobilization, Colloids Surf. B Biointerf. 73 (2009) 156-160.

[54] P. Calvez, E. Demers, E. Boisselier, C. Salesse, Analysis of the contribution of saturated and polyunsaturated phospholipid monolayers to the binding of proteins, Langmuir 27 (2011) 1373-1379.

[55] R. Brasseur, J.A. Killian, B. De Kruijff, J.M. Ruysschaert, Conformational analysis of gramicidin-gramicidin interactions at the air/water interface suggests that gramicidin aggregates into tube-like structures similar as found in the gramicidin-induced hexagonal HIIphase, BBA - Biomembr. 903 (1987) 11-17.

[56] E.J.S. Claereboudt, I. Eeckhaut, L. Lins, M. Deleu, How different sterols contribute to saponin tolerant plasma membranes in sea cucumbers, Sci. Rep, 8 (2018) 1-11.

[57] L. Lins, R. Brasseur, The hydrophobic effect in protein folding, FASEB J. 9 (1995) 535-540.

[58] T. Parasassi, E. Gratton, Membrane lipid domains and dynamics as detected by Laurdan fluorescence, J. Fluoresc. 5 (1995) 59-69.

[59] F.M. Harris, K.B. Best, J.D. Bell, Use of laurdan fluorescence intensity and polarization to distinguish between changes in membrane fluidity and phospholipid order, Biochim. Biophys. Acta - Biomembr. 1565 (2002) 123-128.

[60] T. Parasassi, G. De Stasio, G. Ravagnan, R.M. Rusch, E. Gratton, Quantitation of lipid phases in phospholipid vesicles by the generalized polarization of Laurdan fluorescence, Biophys. J. 60 (1991) 179-189.

[61] M. Ouberai, et al., The Pseudomonas aeruginosa membranes : A target for a new amphiphilic aminoglycoside derivative ? BBA - Biomembr. 1808 (2011) 1716-1727.

[62] J.E. Ladbury, B.Z. Chowdhry, Sensing the heat: the application of isothermal titration calorimetry to thermodynamic stidies of biomolecular interactions, Chem. Biol. 3 (1996) 791-801.

[63] B. Bechinger, K. Lohner, Detergent-like actions of linear amphipathic cationic antimicrobial peptides, BBA - Biomembr. 1758 (2006) 1529-1539.

[64] M. Bramkamp, D. Lopez, Exploring the Existence of Lipid Rafts in Bacteria, Microbiol. Mol. Biol. Rev. 79 (2015) 81-100.

[65] A. Toledo, et al., Lipid rafts can be form in the inner and outer membranes of
Borrelia burgdorferi and have different properties and associated proteins, Mol. Microbiol. 108 (2019) 63-76.

[66] G. Brezesinski, H. Möhwald, Langmuir monolayers to study interactions at model membrane surfaces, Adv. Colloid Interface Sci. 100-102 (2003) 563-584.

[67] C. Stefaniu, G. Brezesinski, H. Möhwald, Langmuir monolayers as models to study processes at membrane surfaces, Adv. Colloid Interface Sci. 208 (2014) 197-213.

[68] D. Marsh, Lateral pressure in membranes, BBA 1286 (1996) 183-223.

[69] E. Mantil, I. Buznytska, G. Daly, A. Ianoul, T.J. Avis, Role of Lipid Composition in the Interaction and Activity of the Antimicrobial Compound Fengycin with Complex Membrane Models, J. Membr. Biol. (2019), https://doi.org/10.1007/s00232-01900100-6.

[70] S.A. Sanchez, M.A. Tricerri, G. Gunther, E. Gratton, Laurdan Generalized Polarization : from cuvette to microscope, Mod. Res. Educ. Top. Microsc. 1007-1014 (2007).

[71] S. Lebecque, L. Lins, F.E. Dayan, M. Fauconnier, Interactions Between Natural Herbicides and Lipid Bilayers Mimicking the Plant Plasma Membrane, Front. Pharmacol. 10 (2019) 1-11.

[72] T. Kondakova, et al., Glycerophospholipid synthesis and functions in Pseudomonas, Chem. Phys. Lipids 190 (2015) 27-42.

[73] R.N.A.H. Lewis, R.N. Mcelhaney, The physicochemical properties of cardiolipin bilayers and cardiolipin-containing lipid membranes 败, BBA - Biomembr. 1788 (2009) 2069-2079.

[74] R. Arias-cartin, S. Grimaldi, P. Arnoux, B. Guigliarelli, A. Magalon, Cardiolipin binding in bacterial respiratory complexes : Structural and functional implications, BBA - Bioenerg. 1817 (2012) 1937-1949.

[75] C. Arnarez, S.J. Marrink, X. Periole, Identification of cardiolipin binding sites on cytochrome c oxidase at the entrance of proton channels, Sci. Rep. 3 (2013) 1-9.

[76] P. Sperandeo, A.M. Martorana, A. Polissi, Lipopolysaccharide biogenesis and transport at the outer membrane of Gram-negative bacteria, BBA - Mol. celle Biol. lipids 1862 (2017) 1451-1460.

[77] A. Molinaro, M. Newman, R. Lanzetta, M. Parrilli, The Structures of Lipopolysaccharides from Plant-Associated Gram-Negative Bacteria, European J. Org. Chem. 5887-5896 (2009), https://doi.org/10.1002/ejoc.200900682.

[78] G. Erbs, M. Newman, The role of lipopolysaccharide and peptidoglycan, two glycosylated bacterial microbe-associated molecular patterns (MAMPs), in plant innate immunity, Mol. Plant Pathol. 13 (2012) 95-104. 